\title{
Successful Treatment of a Large Coccygeal Pressure Ulcer Using Injectable Acellular Dermal Matrix: A Case Report
}

\author{
Minseo Kim (D), Sungmi Jeon (D), Sang Wha Kim (D) \\ Department of Plastic and Reconstructive Surgery, Seoul National University Hospital, Seoul, Korea
}

\begin{abstract}
Pressure sores are common but troublesome for both patients and clinicians. They can range from mild to severe and must be managed accordingly. Despite advancements in both non-surgical and surgical intervention, no standard treatment protocol has yet been established. Since pressure sores can occur in a variety of clinical settings, treatment must be individualized to the patient's circumstances. Recently, acellular dermal matrix (ADM) has been utilized as an alternative treatment for non-healing wounds. In the present report, we describe the case of a non-ambulatory patient in whom a large pressure sore located near the anus was completely cured using CG paste, an injectable ADM
\end{abstract}

Keywords: Pressure ulcer; Acellular dermis; Wound healing; Conservative treatment

\section{Introduction}

Injectable acellular dermal matrix (ADM) was first introduced in the late 1990s for soft tissue augmentation. As the treatment became more popular, clinicians found that it could also be useful in soft tissue reconstruction. CG paste (CG Bio Inc., Seoul, Korea) is an injectable form of ADM suspended in gelatin for a paste-like consistency. It has been used to treat chronic, non-healing wounds, providing structural support for cell ingrowth and revascularization. Furthermore, it is often preferable to other products because it can be used to fill cavitary wounds [1]. CG paste is stored in a syringe, making it easy to carry and use in outpatient clinics or even at home with simple training.

Most patients with non-healing pressure sores are treated using conservative therapy. However, even when such treatment is given consistently, patients often require surgery eventually [2]. Unfortunately, the medical conditions that cause pressure sores often contraindicate general anesthesia. Previous studies have shown that injectable ADM can be effective in the treatment of pressure sores, but most patients are concurrently treated using negative pressure wound therapy (NPWT), and some with large wounds require additional surgery [3]. Moreover, when the wound is close to the anus, NPWT is difficult to apply as the region has numerous creases and is a humid environment prone to contamination. In the present report, we describe the case of a non-ambulatory patient who recovered completely from a large pressure sore near the anus within a short period. The patient was mainly treated using an injectable ADM.
Case Report

Received: June 22, 2021

Revised: August 10, 2021

Accepted: August 12, 2021

Corresponding author:

Sang Wha Kim, M.D., Ph.D.

Department of Plastic and Reconstructive Surgery, Seoul National University Hospital, 101 Daehak-ro, Jongno-gu, Seoul 03080, Korea

Tel: +82-2-2072-2375

Fax: $+82-2-741-3985$

E-mail: sw1215@snu.ac.kr

This is an Open Access article distributed under the terms of the Creative Commons Attribution Non-Commercia License (https://creativecommons org/licenses/by-nc/4 O/) which permits unrestricted non-commercial use, distribution, and reproduction in any medium, provided the original work is properly cited.

C 2021 Korean Wound Management Society 
The patient provided written informed consent for the publication and the use of his images.

\section{Case}

An 83-year-old male patient was referred to our department for treatment of a $5 \times 3-\mathrm{cm}$, non-healing pressure sore located near the anus. The sore had resulted from the man's non-ambulatory status after surgeries for pyogenic spondylitis, the cause of which was obscure. Previously, the patient had been in World Health Organization (WHO) functional class I, without any underlying disease or surgical history of the spine. A $5 \times 3-\mathrm{cm}$ mild pressure sore, stage 1 , was detected at the coccyx after 2 months of bed rest, during which the man had received intravenous antibiotic treatment for pyogenic spondylitis at another clinic. After transferring to the department of orthopedics of our hospital, the patient underwent two consecutive operations on the spine: interbody fusion and posterior fusion. He was confined to absolute bed rest for 43 days after the two consecutive surgeries, even after rehabilitation therapy.

During the admission period, the pressure sore was well managed by simple dressings with foam dressing material and Repigel ointment (Mundipharma Korea Ltd., Seoul, Korea) and remained stable. After discharge, the patient remained non-ambulatory, and the pressure sore worsened from stage 3 to stage 4 , despite care from a home visiting nurse. Two months after the last spine surgery, the patient visited our outpatient clinic with a $5 \times 3-\mathrm{cm}$ sore, which did not subsequently grow in size (Fig. 1). Simple debridement was performed un-

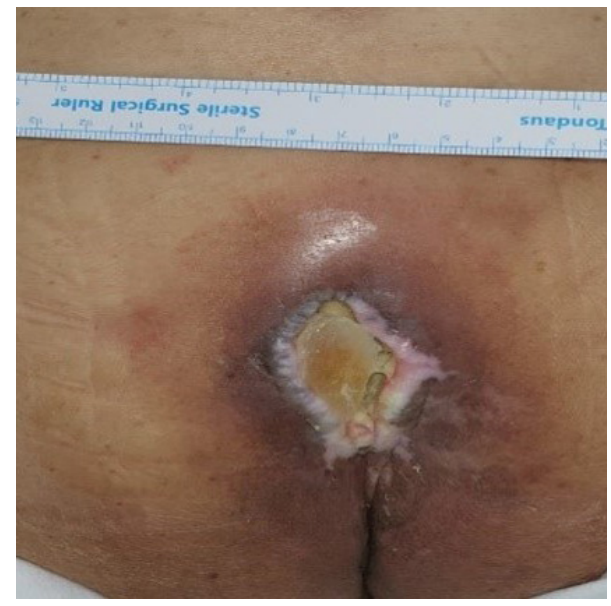

Fig. 1. Medical photo of the wound. Necrotic tissue covering pressure sore. der local anesthesia to treat necrotic eschar at the coccyx. The debridement was performed until vital tissue was visible, and the tissue showed no signs of infection, therefore no additional measures, such as tissue culture or antibiotics administration, were implemented (Fig. 2). During follow-up, the patient complained about the strenuous dressing change regimen and that he could not perform daily activities. For this reason, on the 7th postoperative day, NPWT was applied, but the wound continued to leak because it was close to the anus. While NPWT was applied for 2 months, during most of its application the device continued to leak and was ineffective and the patient had to frequently visit the outpatient clinic. Since the wound did not improve even after application of NPWT, the author started to use injectable ADM. CG paste (1 mg) was used to fill the cavitary wound on a weekly basis, 10 weeks after previous surgery (Fig. 3). Simple dressings using only Mepilex Border (Mölnlycke, Gothenburg, Sweden) was ap-

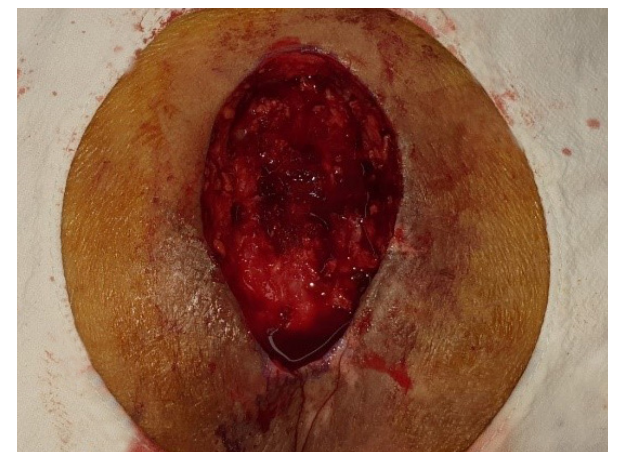

Fig. 2. Intraoperative photo of the wound. Medical photo after simple debridement of the pressure sore.

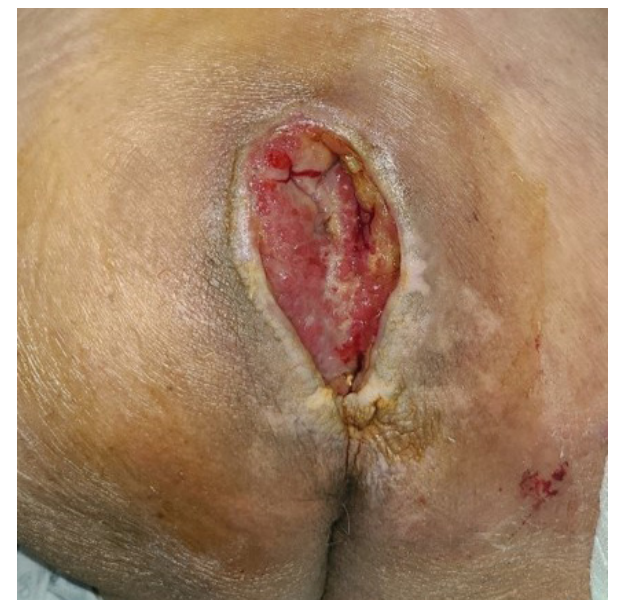

Fig. 3. Medical photo of the wound. Medical photo of the pressure sore before initiation of CG paste application. 
Kim M et al.

Reconstruction using acellular dermis

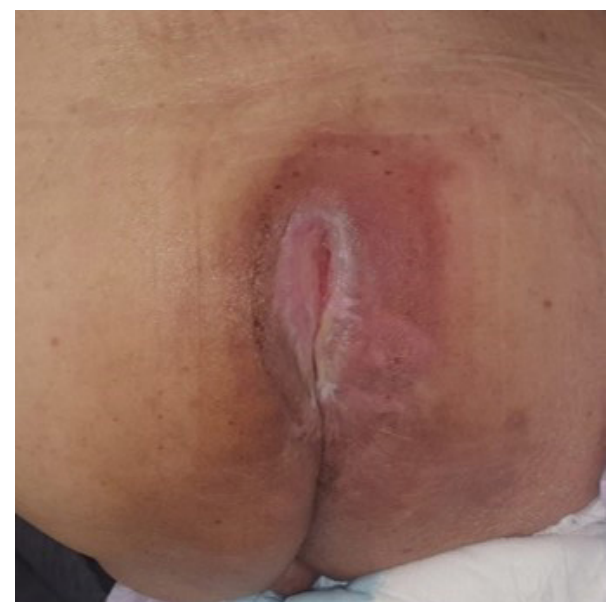

Fig. 4. Medical photo of the wound. Medical photo of the pressure sore after fourth application of CG paste.

plied above the injectable ADM. The dressing did not need to be changed until the next visit, unless the dressing had shifted, since its only role was to hold the material in place. However due to anatomical proximity to the anus and environment of the perineum, the dressing had to be changed almost daily since it became contaminated and fell off constantly. After the sixth application of injectable ADM, the pressure sore completely healed (Figs. 4, 5). Wound re-epithelialization was almost complete, without discharge or odor.

\section{Discussion}

No gold standard management of pressure sores has yet been established; however, a few principles are commonly followed. In terms of patient factors, nutritional repletion is important to reverse protein catabolism and enhance wound healing. In general, $70 \%-90 \%$ of pressure sores are mild and require only conservative therapy. However, in severe cases, more interventions are required, including surgical debridement and reconstruction [4]. Usually, stage 3 or 4 pressure sores indicate surgical reconstruction because there is extensive soft tissue degeneration. Even after spontaneous recovery, pressure sores result in stiff and scanty scar tissue. If the underlying bone is infected, soft tissue coverage supplying adequate blood perfusion is necessary for healing. Long-lasting pressure sores also indicate surgery because they can develop into Marjolin ulcers.

Furthermore, while planning surgery to treat pressure sores, clinicians should evaluate the tolerability of general anesthesia



Fig. 5. Medical photo of the wound. Medical photo of the pressure sore after sixth application of CG paste showing complete recovery.

and postoperative rehabilitation in the patient. Correction of the original disease that caused the pressure sore is recommended before the pressure sore itself is managed, but this is difficult in most cases. In addition, patients must be able to actively participate in rehabilitation therapy. Moreover, the risks of general anesthesia must be balanced with the potential benefits of the operation [5]. In cases of non-ambulatory patients, most pressure sores are severe, and surgical reconstruction is usually considered [6]. However, it is difficult to perform surgery in patients with medical conditions that are associated with high risks under general anesthesia, such as chronic kidney disease and atrial fibrillation. Therefore, treatment with injectable type of ADM can be an attractive option for such patients for it does not impose the risks of general anesthesia to susceptible patients as in our case.

Recent advances have been made in conservative modalities to treat pressure sores. NPWT has been among the most common methods of pressure sore treatment since its introduction in 1997. It increases dermal perfusion, stimulates granulation tissue formation, and reduces edema and bacterial formation. Stage 3 or 4 pressure sores are common indications for NPWT. However, skin irritation, the effort required of the clinician, the need for regular dressing changes, and high costs are commonly overlooked disadvantages of NPWT. More importantly, the device does not always result in consistently beneficial clinical results, and it is usually used as a management strategy before surgical reconstruction $[7,8]$.

Some previous reports have used injectable ADM with other treatments such as NPWT to treat non-healing pressure 
sores. Most severe cases were treated concurrently with NPWT $[1,3,9]$. However, when pressure sores are larger than $2 \times 2 \mathrm{~cm}$, complete re-epithelialization is not achieved, and most patients require additional surgery. In the present case, even though NPWT was applied, it proved ineffective and was inactive during most of its application, and furthermore it was not used concurrently with injectable type of ADM. Injectable ADM was used successively after NPWT proved ineffective. The sore was adjacent to the anus, so defecation, movement, the humid environment of the perineum, and the creases of the region made NPWT almost impossible. Even after successful application, the treatment was useless since leakage occurred frequently. In previous cases which used NPWT with injectable type of ADM, authors reported fall-out of injectable ADM during each dressing change, which can be detrimental to patients with pressure sores located at places anatomically unfit for NPWT as in our case [1]. Our case is unique in that a large-sized pressure sore proximal to the anus was treated completely using mainly injectable ADM without aid of other treatments.

Along with the advantages of an injectable ADM, our team focused on a few previous studies showing that ADMs are resistant to microbial penetration [10], and reports showing that ADMs resist infection that could otherwise develop into serious problems [11]. Our team used an injectable ADM in the present case to overcome the anatomical proximity to the anus and protect against further infection, which is a common problem in pressure sores near the anus.

Pressure sore is a complex medical condition that requires a multi-disciplinary treatment approach because it is difficult to cure. Clinicians should make decisions based on patients' individual situations [2]. The present case demonstrated that injectable ADM could be used to treat large pressure sores near the anus without additional surgery for patients with a high risk of recurrence who cannot tolerate extensive surgery under general anesthesia. We suggest using an injectable ADM as a novel option to treat large, non-healing pressure sores near the anus.

\section{Conflict of interest}

No potential conflict of interest relevant to this article was reported.

\section{ORCID iDs}

Minseo Kim

https://orcid.org/0000-0002-5536-3920

Sungmi Jeon

Sang Wha Kim

https://orcid.org/0000-0001-9823-0469

https://orcid.org/0000-0003-0430-3458

\section{References}

1. Ahn SK, Choi HJ, Lee JB, et al. A clinical study of micronized acellular dermal matrix collagen paste application with negative pressure wound therapy. J Wound Manage Res 2019;15:23-30.

2. Cushing CA, Phillips LG. Evidence-based medicine: pressure sores. Plast Reconstr Surg 2013;132:1720-32.

3. Jeon M, Kim SY. Application of a paste-type acellular dermal matrix for coverage of chronic ulcerative wounds. Arch Plast Surg 2018;45:564-71.

4. Reuler JB, Cooney TG. The pressure sore: pathophysiology and principles of management. Ann Intern Med 1981;94: 661-6.

5. Sorensen JL, Jorgensen B, Gottrup F. Surgical treatment of pressure ulcers. Am J Surg 2004;188(1A Suppl):42-51.

6. Ravi HS, Mehta MA. A study to assess the various factors and treatment modalities of pressure sore in a tertiary care hospital. Int Surg J 2019;6:2324-31.

7. Robert N. Negative pressure wound therapy in orthopaedic surgery. Orthop Traumatol Surg Res 2017;103:S99-S103.

8. Dumville JC, Webster J, Evans D, et al. Negative pressure wound therapy for treating pressure ulcers. Cochrane Database Syst Rev 2015;(5):CD011334.

9. Malmsjo M, Gustafsson L, Lindstedt S, et al. The effects of variable, intermittent, and continuous negative pressure wound therapy, using foam or gauze, on wound contraction, granulation tissue formation, and ingrowth into the wound filler. Eplasty 2012;12:e5.

10. Fahrenbach EN, Qi C, Ibrahim O, et al. Resistance of acellular dermal matrix materials to microbial penetration. JAMA Dermatol 2013;149:571-5.

11. Milburn ML, Holton LH, Chung TL, et al. Acellular dermal matrix compared with synthetic implant material for repair of ventral hernia in the setting of peri-operative Staphylococcus aureus implant contamination: a rabbit model. Surg Infect (Larchmt) 2008;9:433-42. 